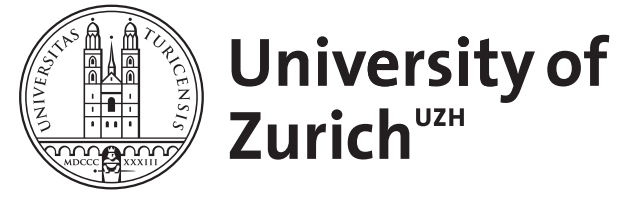
Archive

University of Zurich

University Library

Strickhofstrasse 39

CH-8057 Zurich

www.zora.uzh.ch

Year: 2010

"Modsigelsens Tegn". Eine kleine christologische Semiotik

Bühler, Pierre

DOI: https://doi.org/10.1515/9783110223026.117

Posted at the Zurich Open Repository and Archive, University of Zurich

ZORA URL: https://doi.org/10.5167/uzh-44519

Book Section

Published Version

Originally published at:

Bühler, Pierre (2010). "Modsigelsens Tegn". Eine kleine christologische Semiotik. In: Cappelørn, Niels Jørgen; Deuser, Hermann; Söderquist, K Brian. Kierkegaard's late writings. Berlin, DE: de Gruyter, $117-128$

DOI: https://doi.org/10.1515/9783110223026.117 


\title{
„Modsigelsens Tegn“ \\ Eine kleine christologische Semiotik
}

\author{
Von PIERRE BÜHLER
}

\begin{abstract}
The article deals with a paragraph in Practice in Christianity in which Kierkegaard presents Jesus Christ as a "sign of contradiction". By the same occasion, he develops a brief theory of sign. To understand the signification and function of this "little christological semiotics", the author looks first at the biblical reference of this notion (Luke 2:34f.), tries to situate briefly the topic of contradiction in Kierkegaard's thought and then comments the concerned paragraph. Integrating contradiction into christology allows Kierkegaard to show how the contradiction in Jesus Christ reveals the contradiction in human existence.
\end{abstract}

Wenn man die Einübung im Christentum ${ }^{1}$ mit früheren Werken Kierkegaards vergleicht, fällt auf, dass relativ viele Themen dieses Werkes bereits anderswo behandelt und hier wieder in neuer Manier aufgenommen werden. Da es um Christologie geht, entstehen so unter anderem mehrere Verbindungen zu den Philosophischen Brocken und zur Abschliessenden Unwissenschaftlichen Nachschrift. Ich denke hier vor allem an Aspekte wie etwa: der Gott-Mensch, das schlechthinnige Paradox, die Möglichkeit des Ärgernisses, die Knechtsgestalt, das Inkognito, die Gleichzeitigkeit, die indirekte Mitteilung. ${ }^{2}$ Wenn ich mich nicht täusche, ist jedoch ein Aspekt in EC zu finden, der vorher bei Kierkegaard nicht vorkommt und nachher nur höchst selten wieder aufgenommen wird, und zwar handelt es sich um die knappe Darstellung des Gott-Menschen als „Zeichen“ (auf Dänisch „Tegn“), und genauer als „Zeichen des Wider-

1 Im Weiteren EC abgekürzt; nach GW1 18 zitiert (auf Dänisch: SKS 12). Auch übrige Stellen aus Kierkegaards Werken zitiere ich in der Regel nach GW1. Zu EC, vgl. Hayo Gerdes Sören Kierkegaards, Einübung im Christentum'. Einführung und Erläuterungen, Darmstadt: Wissenschaftliche Buchgesellschaft 1982. Auf den S. 109-138 sind auch Tagebuchaufzeichnungen zu EC zu finden.

$2 \mathrm{Zu}$ diesem Thema vgl. Pierre Bühler „Liebe und Dialektik der Mitteilung“ in Ethik der Liebe. Studien zu Kierkegaards, Taten der Liebe', hrg. v. Ingolf U. Dalferth, Tübingen: Mohr Siebeck 2002, S. 71-87. 
spruchs“ (auf Dänisch „Modsigelsens Tegn“). ${ }^{3}$ Diese kleine christologische Zeichentheorie oder Semiotik möchte ich im Folgenden etwas genauer betrachten und deren Bedeutung im Rahmen von Kierkegaards Werk eruieren.

Für die Auslegung dieser Passage möchte ich in vier Etappen vorgehen: In einem ersten Schritt soll der biblische Bezug der Thematik erläutert werden. Sodann muss kurz überlegt werden, inwiefern der Thematik des Widerspruchs an anderen Stellen in Kierkegaards Werk eine wichtige Rolle zukommt. Im dritten Teil gehe ich dann auf den betreffenden Paragraphen und seine weiteren Bezüge in $E C$ ein und versuche endlich, abschliessend eine kleine Bilanz zu ziehen.

\section{Der biblische Bezug}

Obschon der Bezug bei Kierkegaard eigentlich äusserst diskret behandelt wird, ist er evident: Es geht hier um ein Element aus der Verheissung, die der alte Simeon der Mutter Maria bei der Darbringung Jesu im Tempel verkündet (Luk 2, 34f.):

(34) Und Simeon segnete sie und sagte zu Maria, seiner Mutter: Dieser hier ist dazu bestimmt, viele in Israel zu Fall zu bringen und viele aufzurichten, und er wird ein Zeichen sein, dem widersprochen wird - (35) ja, auch durch deine Seele wird ein Schwert dringen -, damit aus vielen Herzen die Gedanken offenbar werden. ${ }^{4}$

„Zeichen des Widerspruchs“ entstammt der Formel am Ende des Verses 34: „ein Zeichen, dem widersprochen wird“ (auf Griechisch: semeion antilegomenon). Auffallend ist jedoch dass, wenn man in unserem $\S 1$ nach Bezügen zu dieser Bibelstelle sucht, kein explizites Zitat des semeion antilegomenon zu finden ist. Nur im Sinne eines Hinweises auf die Bibelstelle, aber ohne genaues Zitat, wird in $\S 1$ gesagt: „Und in der Schrift heisst der Gott-Mensch ein Zeichen des Widerspruches [...]." Dieser Teil der Bibelstelle selbst wird denn auch nicht kommentiert. Hingegen wird im Schlussteil des Paragraphen eine ausführlichere Auslegung des Schlusses von Vers 35 eingefügt: „damit aus vielen Herzen die Gedanken offenbar werden“. In der Fassung von EC heisst es: „Weil er, antwortet die Schrift,

3 Sie wird in $§ 1$ der „Gedankliche[n] Bestimmung des Ärgernisses, das heisst des wesentlichen Ärgernisses“ entwickelt; EC, 118-121 / SKS 12, 129-132.

4 Zitiert nach Zürcher Bibel 2007, 2. Aufl., Zürich: Theologischer Verlag Zürich, 2008 [2007]. 
den Rat der Herzen offenbaren soll“ (auf Dänisch: „Fordi han, svarer Skriften, skulde gjøre Hjerternes Tanker aabenbare“"). ${ }^{5}$ Es scheint also, dass vom Bibelbezug her Kierkegaard grösseres Interesse an der Auswirkung des Zeichens, an seiner Offenbarungsfunktion hat als am Zeichen selbst.

Dass Kierkegaard mit dieser Stelle nur sehr diskret umgeht, bestätigt sich auch, wenn man im weiteren Werk Kierkegaards nach Hinweisen auf das „Zeichen, dem widersprochen wird“ sucht. Luk 2, 33-40 ist zwar der Bezugstext der zweiten der Zwei erbauliche[n] Reden vom 5. März 1844, ${ }^{6}$ doch wird dort unter der Überschrift „Geduld in Erwartung“ vor allem die Gestalt der Prophetin Hanna in den Versen 36-38 behandelt. An Stellen, wo er auf einzelne Elemente dieser Perikope eingeht, geht es eigentlich immer um den Beginn des Verses 35, nämlich die Ankündigung Simeons, es werde der Mutter Maria ein Schwert durch die Seele dringen. Dieses Motiv des Schwertes hat anscheinend Kierkegaard stark beeindruckt, so dass es sowohl im veröffentlichten als auch im unveröffentlichten Werk wiederholt vorkommt. ${ }^{7}$ In einer Tagebuchaufzeichnung ${ }^{8}$ wird zwischen diesem Schwertmotiv und der Offenbarung der Gedanken in den Herzen ein Bezug hergestellt, was diese Stelle in die Nähe unseres Paragraphen bringt: „Ein Schwert wird durch deine Seele dringen - und des Herzens Gedanken offenbar machen, auch die deinen, ob du noch zu glauben wagst, noch demütig genug bist zu glauben, dass du in Wahrheit die Erwählte unter den Weibern seist, die, welche Gnade gefunden hat vor Gott.“

Auch in einer weiteren Tagebuchaufzeichnung ${ }^{9}$ wird auf den Schluss von Luk 2, 35 Bezug genommen. Kierkegaard betont hier, dass Christus dadurch die Gedanken in den menschlichen Herzen offenbare, dass er die Gnade, die frohe Botschaft, dass alles Gnade sei, verkündige. Die Verkündigung des Gesetzes zwinge die Menschen und stifte so in ihnen den Versuch, sich vor dem Gesetz zu verbergen. Die Gnade jedoch befreie die Menschen vor aller Hemmung. ,Gerade vor der ,Gnade’ gelingt es einem recht zu sehen, was zutiefst im Menschen wohnt.“10

Im späteren Werk Kierkegaards kommt dann nur noch einmal ein Bezug auf das „Zeichen des Widerspruchs“ (ohne Lukas-Zitat!), in Zur

$5 E C, 120 \mathrm{f} . / \mathrm{IC}, 131$.

6 2R44, 119-140.

7 Vgl. etwa $P B, 31 ; E R G, 268 ; C R, 106 ; Z S, 5$; Pap. X 3 A 57; Pap. XI 1 A 40.

8 Pap. XI 1 A 45 (auf Deutsch vgl. T, 5, 173f.).

9 Pap. X 5 A 7.

10 „Just lige over for „Naaden' faaer man ret at see, hvad der boer dybest i et Menneske." 
Selbstprüfung der Gegenwart anbefohlen, und zwar an einer Stelle, wo es darum geht, dass es ein Missverständnis sei, wenn in der Kunst versucht werde, Jesus Christus darzustellen: „, [...]denn dergestalt lässt er sich denn unmöglich darstellen, da seine Herrlichkeit die unsichtbare, die innerliche ist und er, das Zeichen des Widerspruches - welch ein Widerspruch, dies malen zu wollen! - unter einem entgegengesetzten Äusseren sich verbirgt. ${ }^{\text {"11 }}$ Es ist ein Widerspruch in sich, das Zeichen des Widerspruchs zu malen, und das hat damit zu tun, wie wir noch sehen werden, dass das Zeichen des Widerspruchs die Unmittelbarkeit einer künstlerischen Darstellung verunmöglicht.

\section{Bezüge zur Thematik des Widerspruchs an anderen Stellen von Kierkegaards Werk}

Auch wenn der Terminus „Zeichen des Widerspruchs“ sonst im Werk Kierkegaards höchst selten vorkommt, heisst das noch lange nicht, dass die Thematik des Widerspruchs an sich unbedeutend wäre, ganz im Gegenteil. Für Kierkegaard ist Widerspruch die konstitutive Bedingung der Existenz überhaupt: „Solange ich lebe, lebe ich im Widerspruch, denn das Leben selbst ist Widerspruch." ${ }^{12}$ Deshalb kann Kierkegaard auch betonen: „Diejenige Betrachtung, die die Duplizität des Lebens (den Dualismus) sieht, ist höher und tiefer als diejenige, die nach der Einheit sucht $[\ldots]^{\text {“13 }}$

Das führt ihn zu einer kritischen Beurteilung des Identitäts-Prinzips, im Gegensatz zum Kontradiktions-Prinzip, das er als zentral betont. In der bereits zitierten Tagebuchaufzeichnung JJ:261 kommt das eindeutig zur Sprache. Man könne zwar leicht einsehen, dass das Identitäts-Prinzip in gewissem Sinne höher sei und dem Kontradiktions-Prinzip zugrunde liege. Aber es markiere doch nur die Grenze für das menschliche Denken, „wie die blauen Berge, wie die Linie, die der Zeichner den Grund nennt - die Zeichnung ist die Hauptsache." Deshalb: „So lange ich in der Zeit lebe, ist das Identitäts-Prinz. nur eine Abstraktion." Man wird also dem Leben

11 ZS, $151 \mathrm{f}$.

12 JJ:261. Ich zitiere im Folgenden die Tagebuchaufzeichnungen nach der deutschen Edition: Deutsche Søren Kierkegaard Edition, Bd.2: Journale und Aufzeichnungen. Journale $E E-F F-G G-H H-J J-K K$, hrg. von Richard Purkarthofer und Heiko Schulz, Berlin / New York: Walter de Gruyter 2008 (zitierte Stelle: S. 230).

13 JJ:194 (S. 209). 
unter der Bedingung der Zeitlichkeit mit dem Identitäts-Prinzip nie gerecht.

Das hat zur Folge, dass an vielen Orten im Werk Kierkegaards der „Grundsatz vom Widerspruch“ (auf Dänisch: Modsigelsens Grundsaetning) oder das „Kontradiktions-Prinzip“ (auf Dänisch: ContradictionsPrincip) entscheidend zum Tragen kommt, weil diese Perspektive des Widersprüchlichen allein der konkreten Wirklichkeit der Existenz entspricht.

Diese Widersprüchlichkeit gehört auch fundamental zur anthropologischen Konstitution des Menschen, wie sie sowohl im Begriff Angst als auch in der Krankheit zum Tode formuliert wird. Wenn es dort heisst, dass der Mensch eine Synthese sei von Unendlichkeit und Endlichkeit, von Freiheit und Notwendigkeit, von Leib und Seele, usw., dann ist mit dem Begriff „Synthese“ (auch auf Dänisch: Synthese) nicht gemeint, dass sich diese Polaritäten in eine höhere Einheit hinein auflösen lassen. Von der Hegelschen Philosophie her könnte der Begriff „Synthese“ missverständlich wirken, im Sinne einer möglichen Versöhnung der Gegensätze. Doch geht es hier viel eher, wie im obigen Zitat, um eine „Betrachtung, die die Duplizität des Lebens (den Dualismus) sieht“. Das heisst: die beschriebenen Synthesen sind hier als unaufhebbare Gegensätze, grundsätzliche Spannungen der conditio humana betrachtet, in denen sowohl Angst als auch Verzweiflung zum Tragen kommen.

Interessant ist weiterhin, dass der Aspekt des Widerspruchs ebenfalls in der Definition der Komik eine wichtige Rolle spielt, wie es etwa sehr lakonisch in einer Tagebuchaufzeichnung formuliert wird: „Der Widerspruch ist eigtl. die Kategorie des Komischen."14 Im Rahmen einer ausführlichen Bestimmung der Grenzbereiche zwischen den Existenzsphären wird Johannes Climacus in der Abschliessenden unwissenschaftlichen Nachschrift diese Thematik des Widerspruchs als Kategorie des Komischen in den Gestalten der Ironie und des Humors vertiefen und präzisieren. $^{15}$

Zusammenfassend können wir also Folgendes formulieren: Widerspruch erscheint hier als eine Grundbestimmung der menschlichen Existenz als eines „Lebens im Widerspruch“. Das hat seine Entsprechung in der

14 JJ:9 (S. 151). So auch ganz knapp in JJ :276 (S. 235): „,das Komische liegt ständig im Widerspruch."

15 Vgl. dazu ausführlicher Pierre Bühler „Warum braucht das Pathetische den Humor? Humor und Religiosität bei Johannes Climacus“ in Kierkegaard Studies: Yearbook 2005, S. 153-174. 
anthropologischen Konstitution des Menschen als „Synthese“ von Gegensätzen, in denen der Mensch spannungsvoll mit sich selbst verwickelt wird, in Angst und Verzweiflung. Dabei wird er aber auch immer aus der Perspektive des Komischen erprobt, indem Ironie und Humor ihm scherzhaft-ernst das Widersprüchliche in seinem Leben enthüllen.

Was hier existential-anthropologisch erläutert wird, wird nun in unserer „kleinen christologischen Semiotik“ in EC christologisch verarbeitet. Wir wollen nun genauer betrachten, wie das geschieht.

\section{3. „Der Gott-Mensch“ als „Zeichen des Widerspruchs“}

Der $\S 1$ kann in drei Teilen gegliedert werden, die hier der Reihe nach kommentiert werden sollen: Zunächst behandelt der Autor allgemein die Frage „Was ist zu verstehen unter einem Zeichen?" (S. 118). In einem zweiten Schritt (S. 119f.) wird dann der Aspekt des Widerspruchs aufgenommen und deshalb erörtert, in welchem Sinne „Zeichen des Widerspruchs" zu verstehen sei. Schliesslich (S. 120f.) stellt sich die Frage, warum der Gott-Mensch Zeichen des Widerspruchs ist. Diese Warum-Frage wird, wie schon beobachtet, durch eine Kommentierung des Schlusses von Lukas 2, 35 beantwortet: „Weil er, antwortet die Schrift, den Rat der Herzen offenbaren soll.“

Wie das ganze Buch $E C$ steht auch dieser $\S 1$ im Zeichen einer polemischen Abgrenzung sowohl gegenüber einer vom Idealismus geprägten Interpretation des Gott-Menschen als einer spekulativen, fantastischen Einheit von Gott und Mensch, die eine Abstraktion ist und nur unter der Form des Ewigen existiert hätte ${ }^{16}$ als auch gegenüber der dänischen Christenheit, in der man das Christentum abgeschafft hat, indem man es zur Lehre gemacht hat und den Mitteilenden ausgeschaltet hat. ${ }^{17}$ Diese Auseinandersetzungen müssen bei unserer Auslegung natürlich mit berücksichtigt werden; sie bleiben jedoch eher im Hintergrund.

Zugleich muss betont werden, dass der Gedankengang - vielleicht gerade wegen dieses polemischen Akzentes, aber auch, weil der Stil etwas meditativ ist - teilweise relativ redundant ist, und die Thematik in

16 Dabei denkt er an religionsphilosophische Überlegungen von Hegel, aber wohl auch von David Friedrich Strauss.

17 Das ganze Buch EC steht im Zeichen einer Auseinandersetzung mit Mynster und Martensen (vg. dazu Hayo Gerdes Einführung, S. 61-65). 
Schlaufen mit sukzessiven Präzisierungen progressiv vertieft. Deshalb wird hier zum Teil systematisierend vorgegangen.

\section{a) Was ist ein Zeichen?}

Auf diese Frage wird zunächst thetisch geantwortet: „Ein Zeichen ist die verneinte Unmittelbarkeit, oder das zweite Sein, unterschieden vom ersten Sein.“ (118) Damit wird gleich die Verweisungsstruktur des Zeichens als grundlegend hervorgehoben: Es wird von einem Ersten auf ein Zweites Bezug genommen. Damit verbunden, wird die Abweisung der Unmittelbarkeit im Zeichen betont. Wie wir noch sehen werden, kommt mit diesem zweiten Aspekt von Anfang an ein wichtiges Anliegen Kierkegaards zum Zuge: die Ablehnung der unmittelbaren Mitteilung in christologischer Perspektive. Diese Definition wird anhand des Beispiels einer Seemarke erläutert. Sie ist unmittelbar auch etwas, bestehend aus materiellen Dingen wie einer Stange, einem Licht, usw., aber das macht noch nicht ihre Zeichenhaftigkeit aus: „dass sie ein Zeichen ist, ist etwas andres, als was sie unmittelbar ist" (ebd.) Mit den Worten des ersten Satzes könnte man sagen: das „erste Sein“ der Seemarke verweist auf ein „zweites Sein“, und nur dann wird sie zum Zeichen. Diese Grunddimension des Zeichens kann Kierkegaard auch an verschiedenen Stellen des Paragraphen als „Reflexionsbestimmung“ bezeichnen: ,wo ich etwas Auffallendes sehe und es ein Zeichen nenne, ist die Bestimmung durch Reflexion eingetreten“ (ebd.). Darin liegt auch, wie Kierkegaard betont, der Grund für alle mögliche „Irreführung durch Zeichen“: „, das Zeichen ist nur für den, der weiss, dass es ein Zeichen ist, und, streng genommen, nur für den, der weiss, was es bedeutet; für jeden andern ist das Zeichen das, was es unmittelbar ist“" (ebd.). Damit stellt sich natürlich die Frage, von welchen Bedingungen die Verweissungsfunktion des Zeichens abhängig ist. Sicher ist auf jeden Fall, dass es unmittelbar Auffallendes sein muss, das die Aufmerksamkeit auf sich zieht, aber zugleich diese Aufmerksamkeit auf anderes, nicht unmittelbar Gegebenes verweist. Die Gefahr könnte natürlich sein, dass das Zeichen zu seinem Gegenteil wird, das Kierkegaard als ,schlechthinnige Verhülltheit“ (119) bezeichnet. Entweder so, dass das Zeichen unmittelbar gar nicht existiert, oder so, dass es nicht auf anderes verweist, sondern stattdessen im unmittelbaren Sein stecken bleibt. Dieser Aspekt wird nun vertieft, indem die Widerspruchsdimension eingeführt wird. Wie der Autor selber in der Anmerkung zum Paragraphen sagt: „ein Zeichen sein ist schon eine Reflexionsbestimmung, geschweige Zeichen des Widerspruches sein" (121). 


\section{b) In welchem Sinne ist der Gott-Mensch ein Zeichen des Widerspruchs?}

Ein Zeichen des Widerspruchs ist „ein Zeichen, das in sich einen Widerspruch enthält" (ebd.). Dieser Widerspruch besteht nicht einfach darin, dass es da etwas Unmittelbares gibt, das aber zugleich auf anderes verweist: Das ist ja die übliche Struktur des Zeichens. Deshalb präzisiert der Autor seine Definition: ein Zeichen des Widerspruchs ist „ein Zeichen, das in seiner Zusammensetzung einen Widerspruch enthält" (ebd.). Um hier zu einer grösseren Klarheit zu kommen, greift Kierkegaard auf das Beispiel einer „Mitteilung, welche Einheit von Scherz und Ernst“ ist. Das ist für uns ein interessantes Beispiel, in Anbetracht dessen, dass der Widerspruch auch wesenhaft zur Bestimmung des Komischen gehört. Diese Mitteilung muss durch ein unmittelbares Etwas die Aufmerksamkeit auf sich ziehen. Damit ist aber die Zeichenfunktion noch nicht gewährt. Es könnte der Scherz oder der Ernst schlechthin überwiegen, und dann bliebe es bei einer unmittelbaren Mitteilung, entweder von reinem Scherz oder eben von reinem Ernst. Es könnte auch diese Einheit von Scherz und Ernst zu einer reinen „Narrheit" verwandelt werden, und dann fände überhaupt keine Mitteilung statt. Deshalb muss mit dem unmittelbaren Auffallen und Aufmerksammachen zugleich der Übergang zu einer anderen Dimension gewährleistet werden: Es ist „,für den Empfänger unmöglich, unmittelbar zu sagen, was es sei, eben weil der Mitteilende unmittelbar weder Scherz noch Ernst mitteilt" (ebd.). Somit wird der Empfänger herausgefordert, auf der zweiten Ebene „selbsttätig“ zu werden, wie Kierkegaard sagt, also sich dem Widerspruch im „Zweiten Sein“ zu stellen und ihn auszutragen, ihn zu bestehen. Das führt nun Kierkegaard zu einer nochmaligen Definition: „Ein Zeichens des Widerspruchs ist etwas, das die Aufmerksamkeit auf sich zieht und alsdann, indem die Aufmerksamkeit sich gegen es richtet, sich als einen Widerspruch enthaltend erweist" (ebd.).

Mit dem Hinweis darauf, dass die Schrift den Gott-Menschen Zeichen des Widerspruchs nennt (impliziter Hinweis auf Luk 2, 34!), kann er nun diese Kategorie christologisch erörtern. Zunächst grenzt er sich scharf von der Auffassung des Gott-Menschen als spekulativer Einheit ab, denn da wäre der Widerspruch verloren. Entscheidend ist hier, dass der GottMensch ein einzelner Mensch ist, denn nur dann kann „der grösstmögliche, der qualitative" (120) Widerspruch stattfinden. Daraus folgt diese entscheidende Stelle (ebd.): „Zeichen des Widerspruchs sein heisst, etwas andres sein, das in Widerstreit steht zu dem, was man unmittelbar ist. So mit dem Gott-Menschen. Unmittelbar ist er ein einzelner Mensch, ganz wie 
andre Menschen, ein geringer unansehnlicher Mensch; aber nun der Widerspruch, dass er Gott ist."

Damit ist die Verweisungsstruktur im christologischen Zeichen des Widerspruchs klar gekennzeichnet. Wie kann nun aber dieser einzelne, unansehnliche Mensch Aufmerksamkeit auf sich ziehen? Kierkegaard erwähnt kurz zwei Möglichkeiten: „Hierzu dient wesentlich das Mirakel, oder ein vereinzelter unmittelbarer Ausspruch, dass er Gott sei“ (ebd.). Könnte das nicht den Widerspruch aufheben, wenn hier in diesem Sinne unmittelbare Mitteilungen stattfinden? Kierkegaard sieht diese Schwierigkeit. Das Mirakel und der vereinzelte unmittelbare Ausspruch sind nicht schlechthin unmittelbare Mitteilung, denn was sie aussagen steht in radikalem Kontrast zu dem, was man unmittelbar sieht, den unansehnlichen Menschen. Die Unkenntlichkeit, das Inkognito des Gott-Menschen als eines unansehnlichen Menschen, sorgt dafür, dass das Aufmerksammachen des Mirakels oder des vereinzelten unmittelbaren Ausspruchs sich nicht in unmittelbare Mitteilung auflöst. Gerade dadurch schaffen sie Aufmerksamkeit, dass sie auf eine andere Perspektive verweisen. Angesichts dessen, was unmittelbar wahrgenommen wird, stiften sie beim Empfänger die Möglichkeit des Ärgernisses oder werden Gegenstand des Glaubens.

\section{c) Warum ist der Gott-Mensch ein Zeichen des Widerspruchs?}

Wie wir schon gesehen haben, antwortet der Autor auf diese Frage mit einem Kommentar zum Schluss von Luk 2, 35. Zunächst polemisiert er mit Ironie gegen „das Moderne“: Weil dieses moderne Denken den GottMenschen zu einer fantastischen Einheit macht, geht in ihm alles ,so unmittelbar wie das Bein in den Strumpf“ (ebd.). Gegen diese grundsätzliche Erleichterung muss betont werden, dass Christus nicht einfach „ein Dozent, der unmittelbar lehrt für Wortmacher, oder Paragraphen für Schnellschreiber diktiert“, ist. „O, es ist so behaglich, Hörer oder Nachschreiber zu sein, wenn alles so ganz unmittelbar geht". Aber, Kierkegaards Warnung ist klar: „, die Herren Hörer und Nachschreiber mögen sich wohl hüten - ihrer Herzen Rat ist es, der offenbart werden wird“ (ebd.). Und damit ist er nun beim Christlichen im strengen Sinne, das Zeichen und seine Wirkung beim Empfänger: ,das Zeichen des Widerspruches, das den Rat der Herzen offenbart" (ebd.).

Wie geht das vor sich? Die Antwort setzt beim Sehen ein, und zwar interessanterweise in einem doppelten Sinn: „Da ist etwas, das macht, dass man es nicht lassen kann, hinzusehen - und sieh, indem man sieht, sieht 
man wie in einen Spiegel, man gelangt dazu, sich selber zu sehen" (121). Die Metapher des Spiegels ist hier ein wichtiges Motiv: Der Gott-Mensch als Zeichen des Widerspruchs eröffnet Selbsterkenntnis des Empfängers. Mit anderen Worten kann man sagen: Die Verweisung verweist auf den Empfänger zurück, indem im Widerspruch des Gott-Menschen dem Menschen durch eine Widerspiegelung offenbar wird, wer er ist. Kierkegaard versucht eine neue Formulierung, nochmals das Bild des Spiegels aufnehmend: „Ein Widerspruch, einem Menschen unmittelbar gegenübergestellt - und wenn man ihn dazu kriegt, darauf hinzusehen: das ist ein Spiegel; indem der Sehende urteilt, muss es offenbar werden, was in ihm wohnt" (ebd.).

Spannend ist nun aber, dass dieses Sich-Sehen des Menschen im Spiegel, den ihm das Zeichen des Widerspruchs entgegenhält, zugleich begleitet wird durch ein Gesehenwerden: „er, der des Widerspruches Zeichen ist, sieht einem unmittelbar ins Herz, indes man hineinstarrt in den Widerspruch" (ebd.). Sehen ist zugleich Gesehenwerden, und nur dadurch kann der Mensch im Rat, in den Gedanken seines Herzens offenbar werden. Das könnte der Dimension der Gnade entsprechen, die in einer oben bereits zitierten Tagebucheintragung hervorgehoben wurde. ${ }^{18}$ Wie wir gesehen haben, ist das ganze Leben des Menschen Widerspruch. In diesem Sinne könnte man nun sagen: beim Sehen und Gesehenwerden im Zeichen des Widerspruchs wird der Mensch in seinem Widerspruch offenbar. Diese Klärung führt den Menschen vor eine Wahl, in Hinsicht darauf, wie er mit diesem Widerspruch in seinem Leben umgehen will, wie er diesen Widerspruch nicht überwinden, sondern bestehen will, „und indem er wählt, und zugleich in dem, das er wählt, wird er selber offenbar" (ebd.).

\section{Abschliessende Bilanz}

Ich möchte versuchen, abschliessend ein paar Aspekte festzuhalten, die aus dieser kleinen christologischen Semiotik hervorgehen. Was hat Kierkegaard damit für seine Argumentation gewonnen? Im grösseren Abschnitt von $E C,{ }^{19}$ in dem wir stehen, ist es für ihn ein grosses Anliegen, erweisen zu können, dass es in christologischer Perspektive keine unmittelbare Mitteilung geben kann. Unser § 1 leistet sozusagen ein semiotisches

18 Vgl. Fußnote 10 und entsprechende Stelle im Text.

19 EC, 117-140. 
Argument dafür: Wenn es möglich ist, ausgehend von Luk 2, 34f., den Gott-Menschen als Zeichen des Widerspruchs aufzufassen, dann ist die Unmöglichkeit der unmittelbaren Mitteilung qua Zeichenhaftigkeit des Gott-Menschen bereits erwiesen. Ein Zeichen ist immer „Reflexionsbestimmung“, wie Kierkegaard sagt, und deshalb unmöglich unmittelbar, weil es wesenhaft Unmittelbares mit Mittelbarem verbindet. Wie der Widerspruch von Scherz und Ernst, so weist auch der Widerspruch eines unansehnlichen Menschen, der zugleich Gott ist, aus der Unmittelbarkeit heraus.

Diese semiotische Argumentation zugunsten der Unmöglichkeit einer christologischen Unmittelbarkeit fügt sich in den weiteren Gedankenduktus ein und wirkt sich deshalb auch auf die weiteren Paragraphen des Abschnittes aus. Insbesondere finden wir deren Spuren in $\S 3,{ }^{20}$ einem Paragraphen, in dem es ja darum geht, die Unmöglichkeit unmittelbarer Mitteilung nochmals grundlegend zu markieren. In diesem Paragraphen wird insgesamt dreimal das Zeichen des Widerspruchs in Anspruch genommen, um diese Unmittelbarkeit zu brechen.

Auch in $\S 6,{ }^{21}$ der dem Thema „Die unmittelbare Mitteilung versagen heisst ,Glauben" fordern" gewidmet ist, wird das christologische Zeichen des Widerspruchs argumentativ eingesetzt. Hier setzt sich Kierkegaard mit dem Glaubensverständnis der modernen Philosophie auseinander: „die ganze moderne Philosophie hat alles getan um es uns einzubilden, dass Glaube eine unmittelbare Bestimmung ist, das Unmittelbare ist." (136) Dieser Entschärfung des Glaubens setzt Kierkegaard die Forderung entgegen, dem Glauben einen verschärften Sinn zu geben, indem dieser Glaube auf das Zeichen des Widerspruchs ausgerichtet wird und so durch dessen Verweisungsstruktur aus der Unmittelbarkeit herausgerissen wird und zu einer Reduplikationsarbeit in der Existenz hingeführt wird. „Man hat sinnlos vergessen, dass der Lehrer hier wichtiger ist als die Lehre. Überall, wo es geschieht, dass der Lehrer wesentlich mit dazugehört, gibt es eine Verdoppelung [...]“. ${ }^{22}$ Deshalb könnte man sagen: Das Zeichen des Widerspruchs will beim Empfänger die Bewegung des Glaubens auslösen, auch wenn es dafür zugleich die Möglichkeit des Ärgernisses einschärfen muss, denn nur so werden die Gedanken im menschlichen Herzen geklärt, auf eine Wahl hin, in der die Gedanken offenbar werden. Aber das eigentliche Ziel im Zeichen des Widerspruchs ist es, durch die Verwei-

$20 E C, 127-131$.

$21 E C, 135-138$.

22 EC, 117 (im Dänischen für das deutsche „Verdoppelung“: Redupplikation). 
sungsarbeit Glauben zu erreichen. Mit Kierkegaards Worten: „Glaube im geschärften Sinne bezieht sich auf den Gott-Menschen. Aber der GottMensch, das Zeichen des Widerspruches, versagt die unmittelbare Mitteilung - und heischt den Glauben." (136)

Das heisst nun aber, dass die semiotische Verweisungsstruktur im Gott-Menschen eine Rückverweisung auf den Empfänger zur Grundausrichtung hat, im Sinne einer Selbsterkenntnis, die in der Widerspiegelung stattfindet, die durch das Hinschauen auf das Zeichen des Widerspruchs ausgelöst wird. Der Mensch sieht sich und der Gott-Mensch sieht ihn, in all dem, was in ihm wohnt. Wenn das ganze Leben, wie wir sahen, Widerspruch ist, können wir sagen, dass im Spiegel, den das Zeichen des Widerspruchs dem Menschen entgegenhält, der Widerspruch des menschlichen Lebens widergespiegelt wird, und dadurch offenbart, enthüllt wird. Im Zeichen des Widerspruchs sieht sich der Mensch und wird er vom GottMenschen gesehen in seinem Verwickeltsein im Widerspruch. Solches Sehen und Gesehenwerden wirkt klärend. Somit ist es Kierkegaard durch seine kleine christologische Semiotik gelungen, zu zeigen, wie der Widerspruch in Jesus Christus den Widerspruch in der menschlichen Existenz offenbart. In solcher Offenbarung liegt göttliche Gnade, die befreiend wirkt. 